services: a systematic literature review. BMC Health Serv Res 2006; 6: 43.

5. McKie C, Ahmad UA, Fellows S, et al. The 2-week rule for suspected head and neck cancer in the United Kingdom: referral patterns, diagnostic efficacy of the guidelines and compliance. Oral Oncol 2008; 44(9): 851-856.

DOI: 10.3399/bjgp10X515430

\section{Asthma inhalers and colour coding: universal dots}

The importance of colour-coded asthma treatment in patient education is well accepted. Traditionally, reliever medication inhalers are blue in colour and preventer inhalers brown. ${ }^{1}$ This custom is not always followed and the inconsistencies in the colour of inhalers create a lot of confusion.

Marketing of the same medication in different colour inhalers and introduction of new drugs and combinations contributed to this predicament. The confusion created by the introduction of generic salbutamol in UK in orangecoloured inhalers has been previously discussed. ${ }^{2}$ Salbutamol in some countries comes in white inhalers with a blue cap.

The colour coding of caps is never reliable as patients can easily change these stoppers.

Across the world, inhaled corticosteroids (ICS) are available as brown, white, magenta, blue, orange brown, and red inhalers. Long-acting betaagonists (LABA) are now marketed in green, blue, white, and greenish blue containers. The new hydrofluoroalkane propellant-based salbutamol is marketed in red or yellow jackets. Interestingly, their advertisement in a journal asks patients to reach for the 'red' in case of need. Inhaled steroids and LABA combinations were introduced in violet, red, and brown inhalers. So at any point of time a patient can be exposed to a collection of coloured inhalers with total uncertainty of its contents.

Ideally, to have certain colours universally accepted would be most

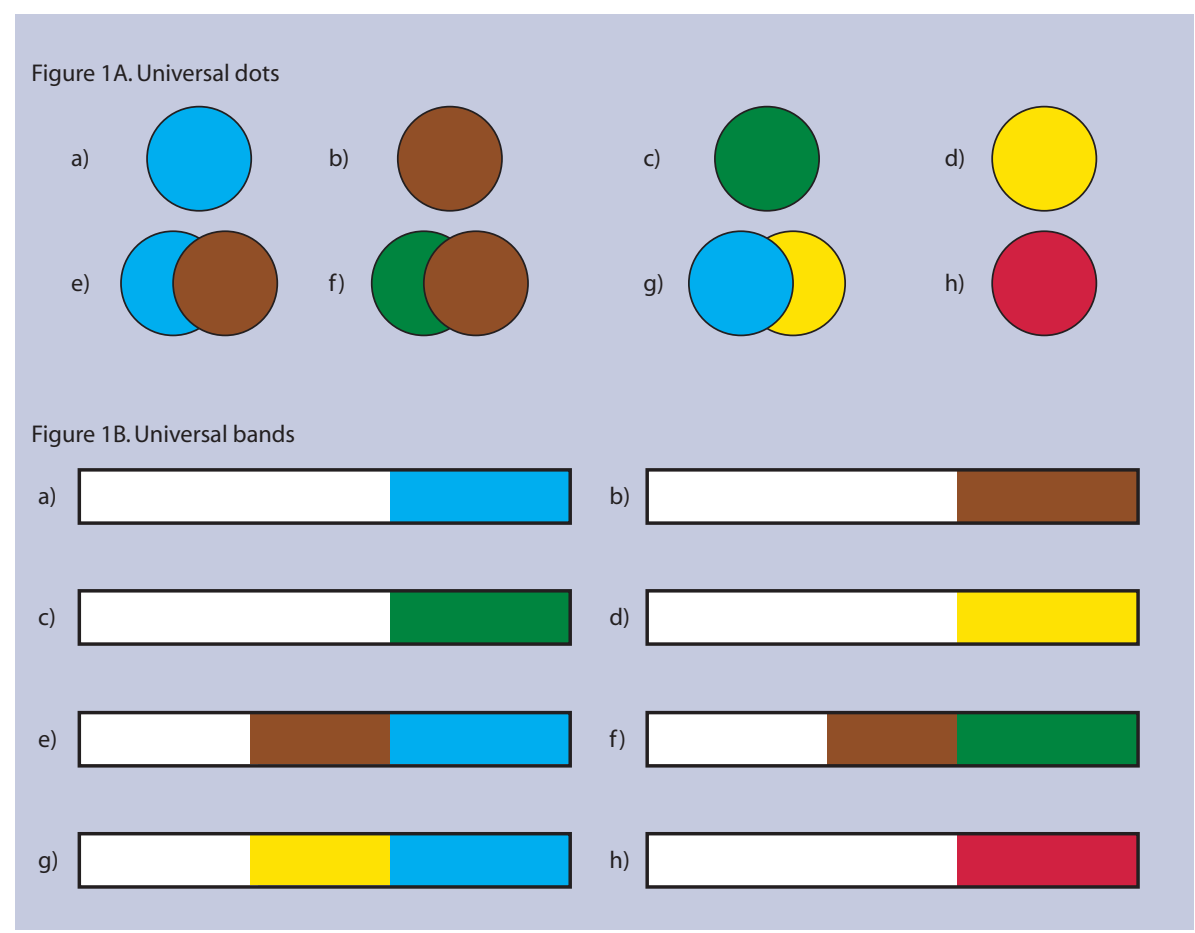

Figure 1A and 1B. a) Blue for beta-agonists. b) Brown for inhaled corticosteroids (ICS). c) Green for long-acting beta-agonists. d) Yellow for anticholinergics. e) Blue and Brown for beta-agonists and ICS combination. f) Green and Brown for long-acting beta-agonists and ICS combination. g) Blue and Yellow for beta-agonists and anticholinergic combination. h) Red to keep in reserve.

effective. But which colour can be used when drugs are marketed in combination? We recommend embedding on the inhalers, small coloured circles or dots. Colours can be assigned to different types of medications. Depending on the contents, the corresponding coloured dot should be placed on the inhaler. When it contains two medications the matching colour dots should partly overlap (Figure 1A). Following the tradition, acceptance will be facilitated by using blue for relievers and brown for inhaled steroids. We suggest green for LABA, yellow for anticholinergics, and black for long-acting anticholinergics. Consequently, one can easily make out that an inhaler with a blue dot contains a bronchodilator irrespective of the fact of where it was purchased, be it in London or in Muscat.

Two overlapping coloured circles, one brown and the other green, convey that the inhaler contains ICS and LABA. Red can be kept aside for future use. We suggest that these identifiers be called ' $U$ dots' (universal inhaler dots). To help patients with visual impairment, ${ }^{3}$ dots should have a slightly raised level with the order of colours reserved universally. Following the same principle, an alternative identification, colour bands, can also be considered; these could be called 'universal bands' or 'U bands' (Figure 1B)

Partridge ${ }^{4}$ narrates his unsuccessful efforts to get responsible bodies interested in the problems created by the introduction of a generic salbutamol inhaler. He is not alone in raising the same concerns. ${ }^{1,5}$ Nevertheless, we hope to revive discussion around the convenience of having uniform colours for inhalers. We call upon all manufacturers to consider this concept and adopt the suggested colours for each class of medications.

\section{B Jayakrishnan,}

Consultant, Department of Medicine, Sultan Qaboos University, PO Box 35, Al Khod, Muscat, Oman.

E-mail: drjayakrish@hotmail.com

Omar A Al-Rawas,

Department of Medicine, Sultan Qaboos University Hospital and College of Medicine and Health Sciences, Oman. 


\section{REFERENCES}

1. Horn CR, Cochrane GM. Colour coding fo bronchodilator inhalers. Lancet 1986; 1(8473): 165

2. Minerva. BMJ 1992; 305(6853): 594

3. Maxwell D. Distinguishing inhalers to aid blind people. BMJ 1992; 305(6863): 1226

4. Partridge M. Coloured inhalers. BMJ 1992; 305(6858): 890.

5. Jones KP. Guidelines on the management of asthma Thorax 1993; 48(10): 1050.

DOI: 10.3399/bjgp10X515449

\section{Steroid treatment cards: patient safety remains at risk}

The British National Formulary advises that all patients prescribed corticosteroids for more than 3 weeks are issued with a steroid treatment card that they carry on their person at all times. ${ }^{1}$ An audit was carried out to determine if these guidelines are being met in patients receiving palliative care at Willow Wood Hospice. A similar survey carried out by Zeppetella in 1998, and published in a letter to this journal, revealed that $43 \%$ of patients referred to St Joseph's Hospice, London, did not have a steroid treatment card, ${ }^{2}$ and I was interested to determine if practice had now improved.

Seventeen patients were included in my audit; all were prescribed corticosteroids orally, either dexamethasone or prednisolone, which they had been taking for over 3 weeks. Nine patients (53\%) did have a card; eight (47\%) did not. Out of those with a card, only two (22\%) admitted to carrying it at all times.

Steroids are prescribed for many indications in palliative care, including the relief of non-specific symptoms, such as anorexia, fatigue, and cachexia syndrome. ${ }^{3}$ However, their long-term usage is associated with serious side effects, such as Cushing's syndrome and an increased susceptibility to infection. Prolonged use may cause suppression of the hypothalamic-pituitary-adrenal axis resulting in adrenal insufficiency with risk of adrenal crisis, cardiovascular collapse, or even death in the event that steroids are stopped suddenly, including during anaesthesia. ${ }^{4}$ The steroid treatment card acts as a reminder to patients about the potential side effects and dangers of abrupt cessation, as well as a warning to healthcare professionals about patients' medication status: ultimately it aims to ensure patient safety.

This audit demonstrated that the British National Formulary guidelines are not being met in 2010: almost 50\% of patients did not have a steroid treatment card and of those with a card, few always carried it. These findings reflect those of Zeppetella's survey, suggesting that this may be a long-standing, widespread problem. Steroid treatment cards should be distributed to and carried by patients on long-term steroids: this issue needs to be recognised and steps taken to improve clinical practice in this area.

\section{Elizabeth Rusby,}

Fourth-year medical student, Low Winsley House, Burnt Yates, Harrogate, HG3 3ES.

E-mail:

Elizabeth.Rusby@student.manchester.ac.uk

\section{REFERENCES}

1. Martin J. British National Formulary 59. London: BM] Group, 2010.

2. Zeppetella G. Steroid treatment cards. Br J Gen Pract 1998; 48(432): 1436.

3. Hardy JR, Rees E, Ling J, et al. A prospective survey of the use of dexamethasone on a palliative care unit. Palliat Med 2001; 15(1): 3-8.

4. Jabbour SA. Steroids and the surgical patient. Med Clin North Am 2001; 85(5): 1311-1317.

DOI: 10.3399/bjgp10X515458

\section{Patients' perceptions of hypertension management: a concern for healthcare practitioners}

Patients' attitudes towards medication have a major impact on disease management, but this is often neglected. A large body of literature has been published from developed nations on the evaluation of non-adherence in chronic disease management and in patients with hypertension in particular. ${ }^{1-2}$ However, there is a paucity of data in the context of hypertensive patients' perspective from developing countries, to gather in-depth information towards hypertension management.

In the context of developing nations like Pakistan, health-seeking behaviour always occurs in the context of medical pluralism, where the patient uses different systems of healing. Patients make independent assessments regarding the use of modern medicines. Orthodox medications do not have such dominance over the population as seen in the western world..$^{3-4}$ In addition, indigenous healing systems, particularly hikmat (treatment with herbs) and spiritual healing, are quite prominent in these populations. There is no legal or official acknowledgment as far as the spiritual healing is concerned; but it is still the treatment of choice for the majority of the population. Insufficient information and understanding emerges as major barriers associated with all aspects of medicine use. Knowledge about the disease and drugs used for its treatment is apparently poor and in return produces a great impact on patients' adherence to medication. Another issue that lies ahead for health practitioners is that discussions of the advantages and disadvantages of drugs in ways that are relevant for individual patients is rarely seen in practice.

What is needed at this point is a complete revision of the practices that are currently being employed in the healthcare system. Individuals must be educated not only on the risk factors, presenting features, and complications of hypertension, but also about the benefits of medications on treatment outcomes and quality of life. Patients may need to be educated about the differences between curing hypertension and treating it with medications. Efforts to educate the public about lifestyle modifications in the prevention of hypertension and other cardiovascular diseases may also be of great importance. Advancement in 